\title{
A carvacrol-thymol blend decreased intestinal oxidative stress and influenced selected microbes without changing the messenger RNA levels of tight junction proteins in jejunal mucosa of weaning piglets
}

\author{
H.-K. Wei ${ }^{1,2}$, H.-X. Xue ${ }^{1,2}$, Z. X. Zhou ${ }^{1,2}$ and J. Peng ${ }^{1,2 \dagger}$ \\ ${ }^{1}$ Department of Animal Nutrition and Feed Science, College of Animal Science and Technology, Huazhong Agricultural University, Wuhan 430070, People's Republic \\ of China; ${ }^{2}$ The Cooperative Innovation Center for Sustainable Pig Production, Wuhan 430070, People's Republic of China
}

Recent studies indicate that intestinal oxidative stress and microbiota imbalance is involved in weaning-induced intestinal dysfunction in piglets. We have investigated the effect of feeding a carvacrol-thymol blend supplemented diet on intestinal redox status, selected microbial populations and the intestinal barrier in weaning piglets. The piglets (weaned at 21 days of age) were randomly allocated to two groups with six pens per treatment and 10 piglets per pen. At weaning day (21 days of age), six piglets were sacrificed before weaning to serve as the preweaning group. The weaned group was fed with a basal diet, while the weaned$C B$ group was fed with the basal diet supplemented with $100 \mathrm{mg} / \mathrm{kg}$ carvacrol-thymol $(1: 1)$ blend for 14 days. On day 7 post-weaning, six piglets from each group were sacrificed to determine intestinal redox status, selected microbial populations, messenger RNA (mRNA) transcript levels of proinflammatory cytokines and biomarkers of intestinal barrier function. Weaning resulted in intestinal oxidative stress, indicated by the increased concentration of reactive oxygen species and thiobarbituric acid-reactive substances present in the intestine. Weaning also reduced the population of Lactobacillus genus and increased the populations of Enterococcus genus and Escherichia coli in the jejunum, and increased mRNA levels of tumor necrosis factor $\alpha(T N F-\alpha)$, interleukin $1 \beta$ and interleukin 6 (IL-6). In addition, decreased mRNA levels of zonula occludens and occludin in the jejunal mucosa and increased plasma diamine oxidase concentrations indicated that weaning induced dysfunction of the intestinal barrier. On day 7 post-weaning, supplementation with the carvacrol-thymol blend restored weaning-induced intestinal oxidative stress. Compared with the weaned group, the weaned-CB group had an increased population of Lactobacillus genus but reduced populations of Enterococcus genus and $\mathrm{E}$. coli in the jejunum and decreased mRNA levels of TNF- $\alpha$. The results indicated that weaning induced intestinal oxidative stress and dysfunction of the intestinal barrier. Dietary supplementation with $100 \mathrm{mg} / \mathrm{kg}$ carvacrol-thymol (1:1) decreased the intestinal oxidative stress and influenced selected microbial populations without changing the biomarkers of intestinal barrier in weaning piglets.

Keywords: weaning, carvacrol, thymol, oxidative stress, intestinal barrier

\section{Implications}

The current study showed that dietary supplementation with a carvacrol-thymol blend decreased the intestinal oxidative stress and influenced selected microbial populations in weaning piglets. However, the carvacrol-thymol blend had limited ability to decrease weaning-induced intestinal inflammation and failed to improve barrier function of the intestine. It therefore has implications for the application of

\footnotetext{
${ }^{\dagger}$ E-mail: pengjian@mail.hzau.edu.cn
}

carvacrol and thymol in the diet of weaning piglets. It also suggested that evaluation of antioxidative, antibacterial and antiinflammatory ability might lead to the development of novel feed additives to promote intestinal health in weaning piglets.

\section{Introduction}

During weaning, piglets suffer social, environmental and dietary stress, all of which contribute to a decrease in their performance. Numerous studies have shown that dysfunction of the intestine, which has important immunological, 
metabolic and barrier functions, has been demonstrated to play a crucial role in weaning-induced growth check (Wijtten et al., 2011; Campbell et al., 2013). Recent studies have indicated that decreased antioxidant activity and increased production of reactive oxygen species (ROS) is involved in weaning-induced intestinal dysfunction, resulting in intestinal oxidative stress (Wang et al., 2008; Degroote et al., 2012; Zhu et al., 2012). The intestinal oxidative stress is frequently associated with inflammation (Rada et al., 2011; Padgett et al., 2013). Elevated proinflammatory cytokines, such as interleukin $1 \beta$ (IL-1 $\beta)$, tumor necrosis factor $\alpha$ (TNF- $\alpha$ ) and interleukin 6 (IL-6), can induce the decreased abundance of tight junction proteins including zonula occludens (ZO-1) and occludin and thus undermine the integrity of the intestinal barrier (Al-Sadi et al., 2009; Suzuki et al., 2011). In addition to ZO-1 and occludin, plasma diamine oxidase has been proposed as a sensitive circulating biomarker for monitoring the intestinal mucosal barrier (Wolvekamp and de Bruin, 1994).

It has been reported that weaning stress dramatically decreases the amount of Lactobacillus and increases the amount of Escherichia coli present in the gut (Franklin et al., 2002; Castillo et al., 2007). A recent study reported that Lactobacillus was negatively correlated with oxidative stress, while, conversely, E. coli showed a strong positive correlation with oxidative stress in the intestines of early weaned piglets (Xu et al., 2014a). In most cases, oxidative stress is characterized by increased generation of ROS which overwhelm the antioxidant capacity, subsequently resulting in damage to cellular macromolecules such as lipids, proteins and DNA. Several kinds of bacteria in the intestines, especially Enterococcus faecalis and E. coli, have been shown to produce extracellular ROS (Huycke and Moore, 2002), whereas Lactobacillus has the ability to inhibit ROS production from fermentation of colon digesta and growth of $E$. faecalis and E. coli (Sun et al., 2010).

Both carvacrol and thymol possess in vitro ROSscavenging activity (Mastelic et al., 2008). In addition, it has been demonstrated that either carvacrol or thymol alone, or in combination, has antibacterial properties to inhibit growth of E. faecalis and E. coli (Michiels et al., 2009; Guarda et al., 2011; Gutiérrez-Fernández et al., 2013). Based on these properties of carvacrol and thymol, we hypothesized that the inclusion of a carvacrol-thymol blend in the diet would scavenge ROS and/or alter the gut microbial populations, such as those of Lactobacillus, E. faecalis and $E$. coli, thus decreasing oxidative stress and preventing weaning-induced dysfunction of the intestinal barrier, and thereby increasing the performance of weaning piglets.

\section{Material and methods}

\section{Animals and experimental design}

Gestating sows (Landrace $\times$ Yorkshire) were fed daily with average quantity of $2.2 \mathrm{~kg}$ of a gestating diet that met the NRC-recommended requirements for nutrients during the period of gestation. After farrowing, sows had free access to a lactation diet that also met NRC-recommended requirements. Each sow nursed 9 to 11 piglets (Duroc $\times$ Landrace $\times$ Yorkshire) before weaning. All sows had free access to water during the gestation and lactation periods.

A total of 120 piglets from 15 litters with an initial BW of $6.5 \pm 0.9 \mathrm{~kg}$ were randomly allocated to two groups with six pens per treatment and 10 piglets per pen. The animals in group 1 were fed with a basal diet (weaned group), while the animals in group 2 were fed with a basal diet supplemented with carvacrol-thymol blend $(50 \mathrm{mg}$ carvacrol and $50 \mathrm{mg}$ thymol/kg of diet) (weaned-CB group) for 14 days. The carvacrol-thymol blend was provided by Novus International Inc. (St. Louis, MO, USA) as Next Enhance $150^{\circledR}(1: 1$, thymol:carvacrol). According to the manufacturer, Next Enhance 150 contains $50 \%$ encapsulated active components (thymol and carvacrol) but no other nutrients. The composition and nutrient levels of the basal diet are shown in Table 1. The feeds used in the current study were prepared weekly and stored in airtight containers. The active components from the feed were analyzed in using gas chromatography MS with an HP 6890 chromatograph (Hewlett Packard, Avondale, PA, USA) connected to an HP 5972 A mass spectrometer (Hewlett Packard) equipped with an HP-5 capillary column $(25 \mathrm{~m} \times 0.25 \mathrm{~mm})$ (Hewlett Packard). The experimental diet contained $51.07 \mathrm{mg} / \mathrm{kg}$ carvacrol and $43.86 \mathrm{mg} / \mathrm{kg}$ thymol, respectively. All of the piglets were given ad libitum access to water and feed. At the weaning day (21 days of age), before separation from the sows, six piglets were sacrificed (preweaning group) to collect jejunal digesta and mucosa, plasma, jejunum and jejunal tissue. When the piglets reached 28 days of age, one median-weight weaned piglet from each pen (total of six piglets/group) was sacrificed to collect plasma, jejunum, jejunal digesta and mucosa. The protocol of the experiment was approved by the Huazhong Agricultural University Institutional Animal Care and Use Committee and performed in accordance with national guidelines. The number of the received ethical clearance is 42000600013283 .

\section{Determination of diarrhea rate and diarrhea index}

The number of pigs with diarrhea was recorded daily throughout the study. The severity of diarrhea was evaluated using the fecal consistency score system (Marquardt et al., 1999). Scores were 0 , firm feces, normal; 1 , pasty, possible slight diarrhea; 2, semi-liquid, definitely unformed feces; or 3, liquid, very watery and frothy diarrhea. The diarrhea rate was calculated as (the total number of pigs with diarrhea/the total number of all experimental pigs) $\times 100 \%$. Diarrhea index was calculated as sum of feces score/total number of pigs.

\section{Sample collection}

At $0800 \mathrm{~h}$ on the day of slaughter, blood was collected in tubes with anticoagulant ethylenediaminetetraacetic acid (EDTA) and plasma was obtained after centrifugation at $3000 \times \mathrm{g}$ for $20 \mathrm{~min}$ at $4^{\circ} \mathrm{C}$ and stored at $-80^{\circ} \mathrm{C}$ until analysis for redox status. Jejunal digesta were collected under sterile conditions and immediately stored at $-80^{\circ} \mathrm{C}$ 
Table 1 Composition and nutrient levels of the basal diet (as-fed basis)

\begin{tabular}{|c|c|}
\hline Ingredients (\%) & Basal die \\
\hline Extruded corn & 214.00 \\
\hline Broken rice & 200.00 \\
\hline Sugar & 50.00 \\
\hline Whey powder & 100.00 \\
\hline Soybean oil & 13.50 \\
\hline Powdered fat & 25.00 \\
\hline Extruded soybean & 125.00 \\
\hline Dehulled soybean meal & 93.80 \\
\hline Fermented bean pulp & 84.00 \\
\hline Spray-dried plasma & 25.00 \\
\hline Dried porcine soluble & 25.00 \\
\hline Limestone & 7.26 \\
\hline Calcium formate & 5.00 \\
\hline Calcium bicarbonate & 14.53 \\
\hline Salt & 1.04 \\
\hline Lysine $(98.5 \%)$ & 2.28 \\
\hline Methionine $(98.0 \%)$ & 1.97 \\
\hline Threonine (98.0\%) & 0.62 \\
\hline Vitamin premix ${ }^{*}$ & 6.00 \\
\hline Trace element premix ${ }^{\dagger}$ & 6.00 \\
\hline \multicolumn{2}{|l|}{ Nutrient composition ${ }^{\ddagger}$} \\
\hline Net energy (kcal/kg) & 2500 \\
\hline $\mathrm{CP}(\%)$ & 19.50 \\
\hline Calcium (\%) & 0.72 \\
\hline Available phosphorus (\%) & 0.40 \\
\hline Digestible lysine (\%) & 1.25 \\
\hline Digestible threonine $(\%)$ & 0.77 \\
\hline Digestible methionine + cystine (\%) & 0.75 \\
\hline
\end{tabular}

*Provided per $\mathrm{kg}$ of diet: vitamin $\mathrm{A} 9600 \mathrm{IU}$, vitamin $\mathrm{D}_{3} 3400 \mathrm{IU}$, vitamin $\mathrm{E}$ $20 \mathrm{mg}$, vitamin $K_{3} 0.30 \mathrm{mg}$, vitamin $B_{1} 1.22 \mathrm{mg}$, vitamin $B_{2} 9.60 \mathrm{mg}$, vitamin $B_{6}$ $2.11 \mathrm{mg}$, vitamin $B_{12} 0.02 \mathrm{mg}$, vitamin $B_{3} 36 \mathrm{mg}$, vitamin $B_{5} 54.30 \mathrm{mg}$, folic acid $0.26 \mathrm{mg}$, biotin $0.15 \mathrm{mg}$, choline chloride $120 \mathrm{mg}$.

${ }^{\dagger}$ Provided per kg of diet: $\mathrm{Zn} 100.05 \mathrm{mg}, \mathrm{Mn} 30.53 \mathrm{mg}$, Fe $100.02 \mathrm{mg}$, Cu $125.28 \mathrm{mg}, 10.29 \mathrm{mg}$, Se $0.32 \mathrm{mg}$. In addition to $1100.05 \mathrm{mg} \mathrm{Zn}$ provided by $\mathrm{ZnSO}_{4}, \mathrm{ZnO}$ was also included in the trace element premix to provide $2250 \mathrm{mg}$ $\mathrm{ZnO} / \mathrm{kg}$ of diet.

${ }^{\mp}$ Nutrient content of diets was calculated using published data for the individual ingredients.

pending the analysis of selected microbial populations. Segments $(5 \mathrm{~cm}$ in length) of mid-jejunum was obtained, opened longitudinally and rinsed thoroughly with chilled physiological saline before fixed in $4 \%$ paraformaldehyde for subsequent histological measurement. In addition, $5 \mathrm{~cm}$ mid-jejunum segments were immediately washed with phosphate-buffered saline and collected for further antioxidant-active and thiobarbituric acid-reactive substances (TBARS) analysis. A third, thoroughly rinsed, sample of jejunum tissue was collected in order to obtain jejunal mucosa on a microscope slide, to determine ROS and to analyze the expression of TNF- $\alpha$, IL-1 $\beta$, ZO- 1 and occludin. The samples were then frozen in liquid nitrogen and stored at $-80^{\circ} \mathrm{C}$.

\section{Determination of jejunal morphology}

The samples were sectioned at $5 \mu \mathrm{m}$ thickness and stained with hemotoxylin and eosin. These were acquired with 100x magnifications using an Olympus BX51 microscope
(Olympus Optical Company, Tokyo, Japan). Intestinal villus height and villus crypt depth were measured using Image-Pro Plus 6.0 image processing and analysis system (Media Cybernetics, Bethesda, MD, USA). For each sample, at least 10 welloriented were measured and the mean value was calculated.

Determination of antioxidant-active and thiobarbituric acidreactive substances in jejunum

Following homogenization of the jejunum tissues in saline solution $(1: 10, w: v)$ and centrifugation at $4000 \times \mathbf{g}$ for $20 \mathrm{~min}$ at $4^{\circ} \mathrm{C}$, supernatants were obtained and analyzed. The activities of superoxide dismutase (SOD) and glutathione peroxidase (GSH-Px) and the contents of TBARS were determined according to the manufacturer's instructions (Nanjing Jiancheng Bioengineering Institute, Nanjing, China) (Wei et al., 2015). SOD activity was determined as its ability to inhibit the reduction of nitro blue tetrazolium. GSH-Px activity was determined based on quantifying the rate of oxidation of GSH to glutathione disulfide (GSSG) by $\mathrm{H}_{2} \mathrm{O}_{2}$, catalyzed by GSH-Px. TBARS were analyzed based on the reaction with 2-thiobarbituric acid, using an Infinite ${ }^{\circledR} \mathrm{M} 1000$ Pro microplate reader (Tecan, Morrisville, NC, USA).

Chemiluminescence measurement of reactive oxygen species Levels of ROS were measured by a chemiluminescence assay using luminol (5-amino-2, 3 dihydro-1, 4-phthalazinedione; Sigma, St. Louis, MO, USA) as the probe. The measurements were made as described by Du et al. (2010), with minor changes to the technique, using a multiple function microplate reader (Mithras LB 940; Berthold Technologies, Bad Wildbad, Germany). Briefly, $50 \mu$ l of plasma or $10 \%$ jejunal mucosa homogenate (tissue weight $(\mathrm{mg}):$ saline $(\mu \mathrm{l})=1: 9$ ) and $20 \mu \mathrm{l}$ of horseradish peroxidase (HRP) (12.4 U of HRP type VI $310 \mathrm{U} / \mathrm{mg}$; Sigma) were added to $150 \mu$ l of KrebsHEPES buffer $(118 \mathrm{mM} \mathrm{NaCl}, 4.7 \mathrm{mM} \mathrm{KCl}, 1.3 \mathrm{mM} \mathrm{CaCl} 2$, $1.2 \mathrm{mM} \mathrm{MgCl} 2,1.2 \mathrm{mM} \mathrm{KH}_{2} \mathrm{PO}_{4}, 25 \mathrm{mM} \mathrm{NaHCO} 3,10 \mathrm{mM}$ $\mathrm{N}$-2-hydroxyethylpiperazine- $\mathrm{N}-2^{2}$-ethanesulfonic acid, $10 \mathrm{mM}$ glucose; pH 7.4). Finally, $50 \mu \mathrm{l}$ of $1.25 \mathrm{mM}$ luminol was added to the mixture, and chemiluminescence was recorded in the dynamic mode. The area under the curve was calculated using Origin 7.0 software (OriginLab Corp., Northampton, MA, USA).

\section{Quantitative $P C R$}

Total RNA was isolated from the jejunal mucosa of the piglets using TRIZOL reagent (Invitrogen, Carlsbad, CA, USA) according to the manufacturer's protocol. The concentration of total RNA was measured using a NanoDrop 2000/C spectrophotometer (Thermo, Rockford, IL, USA). The complementary DNA (CDNA) was reverse-transcribed from $2 \mu \mathrm{g}$ of eluted RNA using a TRANScript M-MLV kit (Toyobo, Shiga, Japan) based on the manufacturer's instructions. Following 10 -fold dilution, cDNA reverse-transcription was performed to establish the relative quantification of gene amplification. The quantitative PCR for TNF- $\alpha$, IL-1 $\beta$, ZO-1 and occludin, as well as $\beta$-actin as internal control, was performed using their respective primer pairs. All the primer pairs were designed 
Wei, Xue, Zhou and Peng

Table 2 Primers used for quantitative $P C R$

\begin{tabular}{|c|c|c|c|}
\hline Target group & Sequence of primers ( $5^{\prime}$ to $\left.3^{\prime}\right)$ & Product size (bp) & Annealing temperature $\left({ }^{\circ} \mathrm{C}\right)$ \\
\hline Lactobacillus genus & $\begin{array}{l}\text { AGCAGTAGGGAATCTTCCA } \\
\text { CACCGCTACACATGGAG }\end{array}$ & 341 & 58 \\
\hline Enterococcus genus & $\begin{array}{l}\text { CCCTTATTGTTAGTTGCCATCATT } \\
\text { ACTCGTTGTACTTCCCATTGT }\end{array}$ & 144 & 61 \\
\hline Escherichia coli & $\begin{array}{l}\text { CATGCCGCGTGTATGAAGAA } \\
\text { CGGGTAACGTCAATGAGCAAA }\end{array}$ & 96 & 60 \\
\hline TNF- $\alpha$ & $\begin{array}{l}\text { CACCACGCTCTTCTGCCTACTG } \\
\text { TTGAGACGATGATCTGAGTCCTTGG }\end{array}$ & 115 & 63 \\
\hline IL-1 $\beta$ & $\begin{array}{l}\text { AAAGGGGACTTGAAGAGAG } \\
\text { CTGCTTGAGAGGTGCTGATGT }\end{array}$ & 286 & 58 \\
\hline ZO-1 & $\begin{array}{l}\text { GGCGCACGGCGAAGGTAATT } \\
\text { CTATCAAACTCAGGAGGCGGCACT }\end{array}$ & 405 & 60 \\
\hline Occludin & $\begin{array}{l}\text { GGAGTGATTCGGATTCTGTCTATGCT } \\
\text { CGCCTGGGCTGTTGGGTTGA }\end{array}$ & 423 & 60 \\
\hline$\beta$-Actin & $\begin{array}{l}\text { CCAGGTCATCACCATCGG } \\
\text { CCGTGTTGGCGTAGAGGT }\end{array}$ & 158 & 60 \\
\hline
\end{tabular}

TNF- $\alpha=$ tumor necrosis factor $\alpha$ IL-1 $\beta=$ interleukin $1 \beta ;$ ZO-1 = zonula occludens.

using Primer5 software package (Primer-E Ltd, Plymouth, UK). The sequence of primers is presented in Table 2. The reaction was performed in a total volume of $20 \mu$ containing $4 \mu \mathrm{l}$ of $\mathrm{CDNA}, 1 \mu \mathrm{l}$ of forward and reverse primers, $10 \mu \mathrm{l}$ of iTaq SYBR Green PCR Master Mix (Bio-Rad, Hercules, CA, USA), and $5 \mu$ of nuclease-free water. The PCR analysis was performed at $95^{\circ} \mathrm{C}$ for $4 \mathrm{~min}$, followed by 40 cycles of denaturation at $94^{\circ} \mathrm{C}$ for $10 \mathrm{~s}$, annealing at a suitable temperature (Table 2) for $10 \mathrm{~s}$ and extension at $72^{\circ} \mathrm{C}$ for $30 \mathrm{~s}$; a product melting curve was then performed to confirm the specificity of amplification. The results were analyzed by the method of $2^{-\Delta \Delta C_{t}}$.

\section{Quantification of selected microbial populations}

Microbial genomic DNA was extracted from jejunal digesta using a QIAamp DNA stool kit (Qiagen, Valencia, CA, USA) in accordance with the manufacturer's instructions. Briefly, to release bacterial cells from the digesta, $2 \mathrm{~g}$ of jejunal digesta was washed three times in saline containing $0.1 \%$ Tween 80 . During each washing, digesta were shaken vigorously by hand for $5 \mathrm{~min}$ and then centrifuged at $4^{\circ} \mathrm{C}$ for a further $5 \mathrm{~min}$. After removal of the pellet, the bacterial cells were recovered from the pooled washing solutions by centrifugation at $27000 \times \mathbf{g}$ for $15 \mathrm{~min}$ at $4^{\circ} \mathrm{C}$. To lyse the bacterial cells, bacterial samples were homogenized in buffer ASL (stool lysis buffer) and heated at $95^{\circ} \mathrm{C}$ for $5 \mathrm{~min}$. Following incubation with an InhibitEx (Qiagen) tablet to remove potential PCR inhibitors, the lysates were treated with buffer $\mathrm{AL}$ and proteinase $\mathrm{K}$ at $70^{\circ} \mathrm{C}$ for $10 \mathrm{~min}$. DNA was precipitated with ethanol and then purified on a QIAamp spin column and eluted in $50 \mu \mathrm{l}$ of $\mathrm{AE}$ buffer (10 mM Tris-HCl, 0.5 mM EDTA, pH 9.0).

Genomic DNA from jejunal digesta was amplified by routine PCR using species- and genus-specific primers (Table 2). After PCR amplification with a Taq DNA polymerase kit (Promega, Madison, WI, USA) and electrophoresis on a 1.5\% agarose gel, PCR products were purified according to the manufacturer's protocol (Omega, Norcross, GA, USA). The purified PCR products were linked to the pMD18-T vector system (Takara, Shiga, Japan) and then transferred to $E$. coli $\mathrm{DH} 5 \alpha$ (Qiagen) for cloning. After checking the size of the cloned inserts with PCR amplification, the extracted plasmids of the positive clones were sequenced commercially to obtain the positive plasmids.

Serial dilutions of these positive plasmids served to generate standard curves using quantitative real-time PCR (Bio-Rad), permitting estimations of absolute quantification based on respective gene copies. Following 10-fold dilution, microbial genomic DNA was subjected to absolute quantitative PCR assay. The reaction was performed in a total volume of $20 \mu \mathrm{l}$ containing $4 \mu \mathrm{l}$ of template DNA, $1 \mu$ of forward and reverse primers, $10 \mu$ of iTaq SYBR Green PCR Master Mix and $5 \mu \mathrm{l}$ of nuclease-free water. The thermal cycling conditions involved an initial denaturation step at $95^{\circ} \mathrm{C}$ for $4 \mathrm{~min}$ followed by 40 cycles of $95^{\circ} \mathrm{C}$ for $10 \mathrm{~s}$, annealing temperature (Table 2) for $10 \mathrm{~s}$ and $72^{\circ} \mathrm{C}$ for $30 \mathrm{~s}$; this was then followed by a product melting curve to confirm the specificity of amplification. The mean threshold cycle values from the triplicate of each sample were used for the calculations. The data are presented as gene copy numbers per gram of jejunal digesta.

\section{Determination of activity of diamine oxidase in plasma}

Diamine oxidase activity in plasma was measured spectrophotometrically, as described by Hou et al. (2012).

\section{Statistical analysis}

All results are presented as the mean \pm SEM. All statistical analyses were carried out using the SAS statistical package (Version 8.1; SAS Institute Inc., Cary, NC, USA). Comparisons among preweaning, weaned and weaned-CB groups were performed using the one-way ANOVA followed by Tukey's post-hoc test. The Student's $t$ test was used to test for growth 
performance in weaned and weaned-CB groups. The $\chi^{2}$ test was used to test for diarrhea rate. Differences were considered significant when $P<0.05$.

\section{Results}

Growth performance, diarrhea rate and intestinal morphology of piglets

There was no difference in average daily gain, average daily feed intake, feed conversion ratio, diarrhea rate or diarrhea index between the weaned and weaned-CB groups (Table 3). The weaned and weaned-CB groups showed reduced $(P<0.05)$ villus height and villus : crypt ratio compared with the preweaning group (Table 4 ). There was also no difference in intestinal morphology between the weaned and the weaned-CB groups (Table 4).

\section{Antioxidant levels in jejunum}

As shown in Table 5, there were significantly $(P<0.05)$ elevated ROS and TBARS levels, raised activity of GSH-Px, and decreased activity of SOD in the jejunum of weaned piglets compared with preweaning piglets. Compared with the weaned group, the weaned-CB group had significantly $(P<0.05)$ increased activity of SOD and GSH-Px, but a reduced concentration of TBARS in the jejunum. Compared with preweaning piglets, the weaned-CB piglets had higher activity of GSH-Px, but lower activity of SOD. The ROS concentration in the jejunum of weaned-CB piglets was significantly $(P<0.05)$ lower than that of weaned piglets.

Table 3 Growth performance and diarrhea rate of weaning piglets fed with basal diet (weaned) or basal diet supplemented with carvacrolthymol blend (weaned-CB)

\begin{tabular}{lcccc}
\hline \hline Item & Weaned & Weaned-CB & SEM & $P$ \\
\hline BW 2 weeks after weaning & 8.32 & 8.51 & 0.28 & 0.46 \\
Average daily gain (g/day) & 138.1 & 150.5 & 8.36 & 0.52 \\
Average daily feed intake (g/day) & 196.9 & 212.7 & 4.73 & 0.17 \\
Feed conversion ratio & 1.50 & 1.43 & 0.07 & 0.65 \\
Diarrhea rate (\%) & 31.21 & 28.06 & 1.70 & 0.35 \\
Diarrhea index & 0.51 & 0.43 & 0.03 & 0.16 \\
\hline \hline
\end{tabular}

All results are presented as mean $\pm \operatorname{SEM}(n=6)$.

Table 4 Jejunum morphology of 21-day-old (preweaning) and 28-dayold piglets fed basal diet (weaned) or basal diet supplemented with carvacrol-thymol blend (weaned-CB)

\begin{tabular}{lccccc}
\hline \hline Item & Preweaning & Weaned & Weaned-CB & SEM & $P$ \\
\hline Villus height $(\mu \mathrm{m})$ & $620.76^{\mathrm{a}}$ & $353.78^{\mathrm{c}}$ & $410.63^{\mathrm{bc}}$ & 31.10 & $<0.01$ \\
Crypt depth $(\mu \mathrm{m})$ & 305.18 & 368.14 & 345.55 & 13.91 & 0.07 \\
Villi : crypt & $2.09^{\mathrm{a}}$ & $0.96^{\mathrm{c}}$ & $1.19^{\mathrm{bc}}$ & 0.13 & $<0.01$ \\
\hline \hline
\end{tabular}

All results are presented as mean $\pm \operatorname{SEM}(n=6)$.

$a, b, c$ Mean values within a row with different superscript letters were significantly different $(P<0.05)$.
There was no difference in the concentration of TBARS in the jejunum of the weaned- $C B$ and the preweaning groups.

\section{Selected microbial populations in jejunal digesta}

The weaned group had significantly elevated populations of Enterococcus genus and $E$. coli and a reduced population of Lactobacillus genus in jejunal digesta, compared with the preweaning group $(P<0.05$; Figure 1$)$. Compared with the weaned group, the weaned-CB group had significantly $(P<0.05)$ reduced counts of Enterococcus genus and $E$. coli and increased counts of Lactobacillus genus $(P<0.05)$. The population of Enterococcus genus in the weaned- $\mathrm{CB}$ group was significantly higher than in the preweaning group. There was no difference in the populations of $E$. coli and Lactobacillus genus in the weaned-CB and the preweaning groups.

Messenger RNA levels of tumor necrosis factor $\alpha$, interleukin $1 \beta$ and interleukin 6 in jejunal mucosa

The piglets in the weaned group showed a significant increase in messenger RNA (mRNA) levels of TNF- $\alpha$, IL-1 $\beta$ and IL-6 in jejunal mucosa, compared with those in the preweaning group $(P<0.05$; Figure 2$)$. Compared with the weaned group, inclusion of carvacrol and thymol in the diet significantly decreased mRNA transcript levels of TNF- $\alpha$ $(P<0.05)$ in jejunal mucosa (Figure 2). The weaned-CB group had a higher mRNA level of IL- 6 than the preweaning group. However, mRNA levels of TNF- $\alpha$ and IL-1 $\beta$ in jejunal mucosa did not differ between the preweaning and the weaned-CB groups.

\section{Messenger RNA levels of occludin and zonula occludens in jejunal mucosa}

There was a significant $(P<0.05)$ decrease in the mRNA levels of occludin and ZO-1 in the jejunal mucosa of weaned piglets, compared with the preweaning piglets (Figure 3a and b). The mRNA levels of occludin and Z0-1 did not differ between the weaned and the weaned-CB groups. The weaned-CB group had a significantly $(P<0.05)$ reduced mRNA level for ZO-1, but not for occludin, compared with the preweaning group.

\section{Activity of diamine oxidase in plasma}

As shown in Figure $3 c$, the activity of diamine oxidase in plasma significantly increased in the weaned group, compared with the preweaning piglets. The weaned-CB group had a higher activity of diamine oxidase in plasma, compared with the preweaning group $(P<0.05)$. There was no difference in the activity of diamine oxidase in plasma between the preweaning and the weaned-CB groups.

\section{Discussion}

Recent studies have indicated that early weaning may induce intestinal oxidative stress, which contributes to intestinal dysfunction (Wang et al., 2008; Zhu et al., 2012). In this study, we have investigated whether supplementing a carvacrol-thymol blend in the diet lowered early 
Wei, Xue, Zhou and Peng

Table 5 Redox status in jejunum tissue of 21-day-old (preweaning) and 28-day-old piglets fed basal diet (weaned) or basal diet supplemented with carvacrol-thymol blend (weaned-CB)

\begin{tabular}{lcccrl}
\hline \hline Item & Preweaning & Weaned & Weaned-CB & SEM & $P$ \\
\hline ROS (RLU/mg protein) & $1466^{\mathrm{b}}$ & $5782^{\mathrm{a}}$ & $2854^{\mathrm{ab}}$ & 583.64 & $<0.01$ \\
SOD (U/mg protein) & $42.45^{\mathrm{a}}$ & $32.14^{\mathrm{c}}$ & $37.09^{\mathrm{b}}$ & 0.67 & $<0.01$ \\
GSH-Px (U/mg protein) & $23.37^{\mathrm{c}}$ & $38.93^{\mathrm{b}}$ & $47.35^{\mathrm{a}}$ & 1.32 & $<0.01$ \\
TBARS (nmol/mg protein) & $0.35^{\mathrm{b}}$ & $0.53^{\mathrm{a}}$ & $0.40^{\mathrm{a}}$ & 0.03 & $<0.01$ \\
\hline
\end{tabular}

ROS = reactive oxygen species; RLU = relative light unit; SOD = superoxide dismutase; GSH-Px = glutathione peroxidase; TBARS = thiobarbituric acid-reactive substances.

All results are presented as mean $\pm \operatorname{SEM}(n=6)$.

a,b,c Mean values within a row with different superscript letters were significantly different $(P<0.05)$
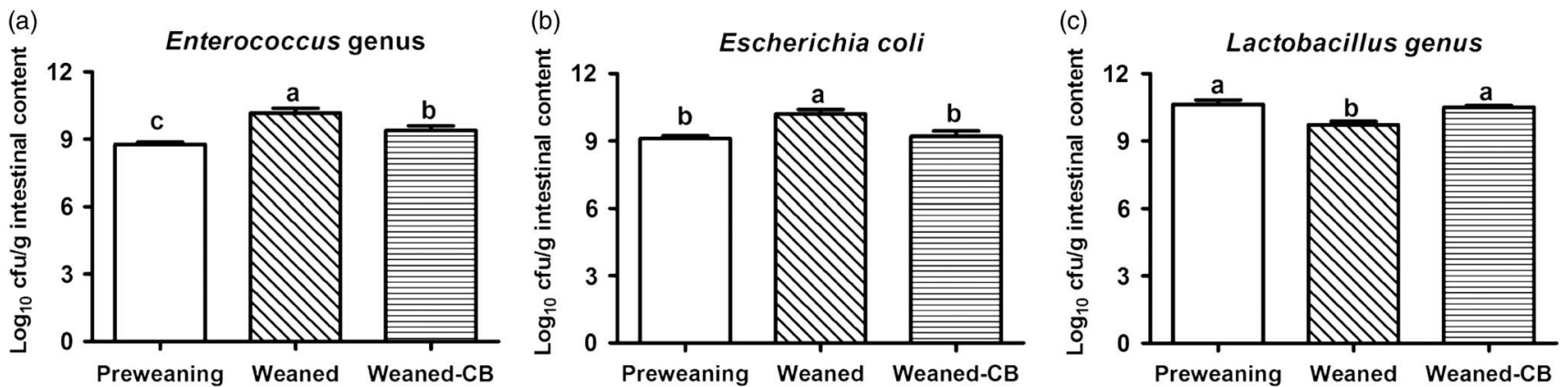

Figure 1 Population of Enterococcus genus (a), Escherichia coli (b) and Lactobacillus genus (c) in jejunum of 21-day-old (preweaning) and 28-day-old (weaned) piglets. Piglets that were 21-day old (preweaning) and 28-day old were fed a basal diet (weaned) or basal diet supplemented with a carvacrolthymol blend (weaned-CB), were sacrificed and then jejunal digesta were collected to determine major microbiota counts. ${ }^{\mathrm{a}, \mathrm{b}, \mathrm{c}}$ Mean values with different superscript letters were significantly different $(P<0.05)$. All results are presented as mean $\pm \operatorname{SEM}(n=6)$.

(a)

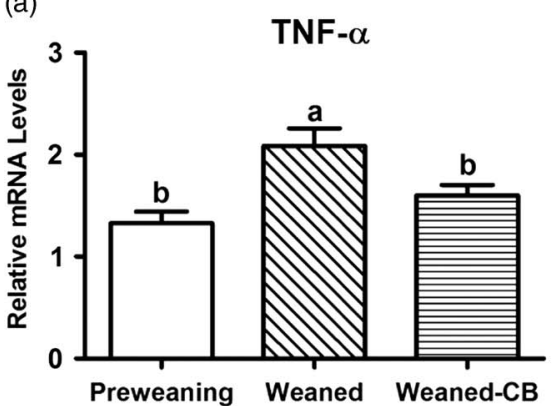

(b)

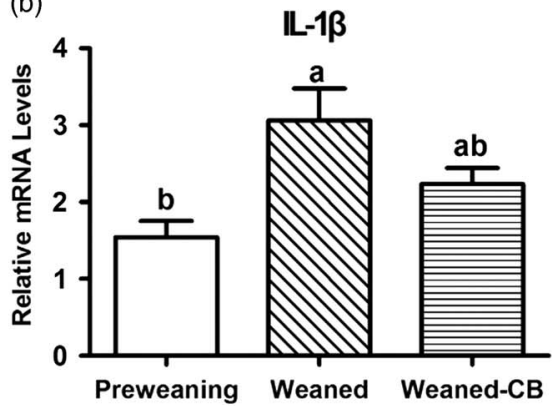

(c)

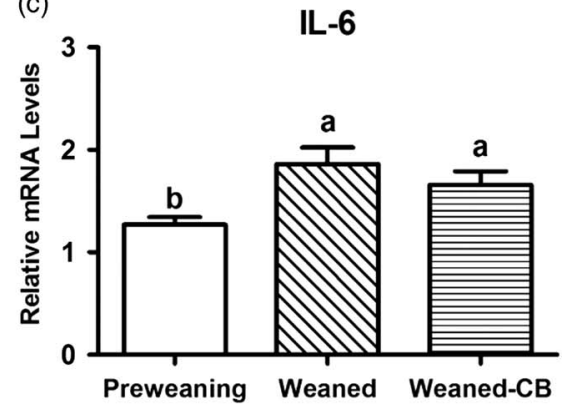

Figure 2 Messenger RNA (mRNA) levels of tumor necrosis factor $\alpha$ (TNF- $\alpha$ ), interleukin $1 \beta$ (IL-1 $\beta$ ) and interleukin 6 (IL-6) in jejunal mucosa of 21-day-old (preweaning) and 28-day-old (weaned) piglets. Piglets that were 21-day old (preweaning) and 28-day old were fed a basal diet (weaned) or basal diet supplemented with a carvacrol-thymol blend (weaned-CB), were sacrificed and then jejunal digest were collected to determine mRNA levels of TNF- $\alpha$, $\mathrm{IL}-1 \beta$ and IL-6. ${ }^{\mathrm{a}, \mathrm{b}}$ Mean values with different superscript letters were significantly different $(P<0.05)$. All results are presented as mean $\pm \mathrm{SEM}(n=6)$.

weaning-induced intestinal oxidative stress and subsequent dysfunction of the intestinal barrier in piglets. The results show that early weaning stress induced intestinal oxidative stress, as indicated by increased levels of ROS and TBARS, as well as decreased activity of SOD and GSH-Px in the jejunum. A diet supplemented with $100 \mathrm{mg} / \mathrm{kg}$ carvacrol-thymol $(1: 1)$ blend reduced weaning-induced oxidative stress. Both carvacrol and thymol have free radical scavenging activity (Mastelic et al., 2008; Archana et al., 2009; Safaei-Ghomi et al., 2009). In the current study, increased activity of antioxidants in the jejunum was observed in the weaned-CB group compared with the weaned group. Therefore, the decreased ROS accumulation in the jejunal mucosa of piglets in the weaned-CB group was at least partly caused by an increased ability to scavenge ROS.

On the other hand, the reduced concentration of ROS in the jejunal mucosa of piglets in the weaned-CB group compared with the weaned group might also have been caused by a reduction in the amount of ROS-producing bacteria (Enterococcus genus and $E$. coli) and ROS-scavenging bacteria (Lactobacillus genus) in the jejunum. It has been demonstrated that intestinal Enterococcus and E. coli produce extracellular ROS (Huycke and Moore, 2002), whereas Lactobacillus has the ability to inhibit ROS 
(a)

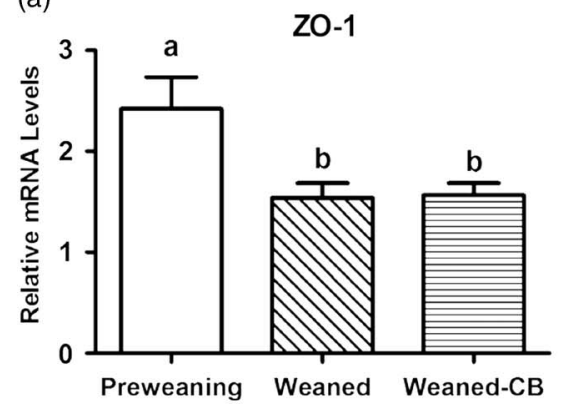

(b)

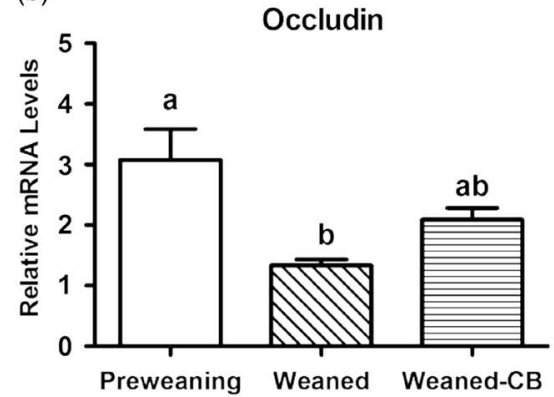

(c)

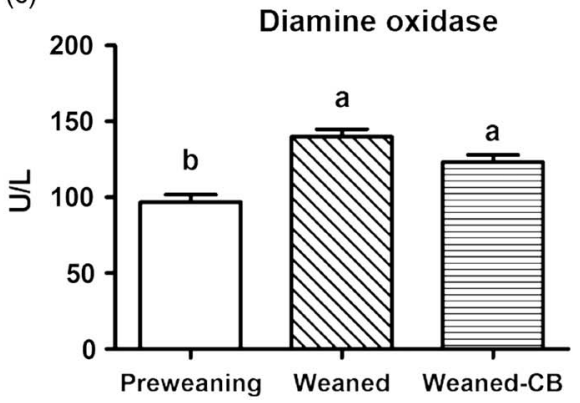

Figure 3 Changes in messenger RNA (mRNA) levels of occludin (a) and zonula occludens (ZO-1) (b) in jejunal mucosa and activity of diamine oxidase (c) in plasma of 21-day-old (preweaning) and 28-day-old (weaned) piglets. Piglets that were 21-day old (preweaning) and 28-day old were fed a basal diet (weaned) or basal diet supplemented with a carvacrol-thymol blend (weaned-CB) and were sacrificed to determine mRNA levels of occludin and ZO-1 in jejunal mucosa and the activity of diamine oxidase in plasma. ${ }^{a, b}$ Mean values within a row with different superscript letters were significantly different $(P<0.05)$. All results are presented as mean $\pm \operatorname{SEM}(n=6)$.

production and the growth of Enterococcus and E. coli (Sun et al., 2010). In this study, the counts of Enterococcus genus and $E$. coli were significantly elevated, and the counts of Lactobacillus genus decreased, following weaning. However, these weaning-induced influences on selected microbial populations were weakened in piglets that consumed a diet supplemented with $100 \mathrm{mg} / \mathrm{kg}$ carvacrol-thymol $(1: 1)$ blend. In fact, it has been demonstrated that dietary supplementation with 100 or $150 \mathrm{mg} / \mathrm{kg}$ essential oils containing $14.5 \%$ thymol and $3.5 \%$ cinnamaldehyde decreases populations of $E$. coli and increases the proportion of Lactobacillus in the ceca of weaning pigs (Li et al., 2012). It seems like as low as $50 \mathrm{mg} / \mathrm{kg}$ carvacrol and thymol or even lower dose affect intestinal microbiota of weaning pigs. In vitro studies have showed that both carvacrol and thymol have antimicrobial activity. However, the minimum inhibitory concentrations (MIC) of carvacrol and thymol against E. coli in vitro are $250 \mathrm{mg} / \mathrm{kg}$ and $375 \mathrm{mg} / \mathrm{kg}$. In addition, MIC of the combination of thymol and carvacrol against strains of E. faecalis is $400 \mathrm{mg} / \mathrm{ml}$ (Guarda et al., 2011). For Lactobacilli genus, in vitro studies showed that carvacrol and thymol only had strong antimicrobial activity at $1000 \mathrm{mg} / \mathrm{ml}$ (Michiels et al., 2009).

Disturbance of gut microbiota is commonly associated with an increased local inflammatory response and dysfunction of the intestinal barrier in weaning piglets (Peace et al., 2011). Our study also found that weaning significantly increased the mRNA levels of proinflammatory cytokines, including TNF- $\alpha, \mathrm{IL}-1 \beta$ and IL- 6 , which is associated with increased biomarker of intestinal permeability (diamine oxidase in plasma) and decreased biomarkers of intestinal tight junctions (ZO-1 and occludin). However, feeding a carvacrol-thymol blend supplemented diet prevented only the weaning-induced TNF- $\alpha$ expression in the jejunum but did not influence the biomarkers of intestinal permeability and tight junctions. In weaning piglets, intestinal inflammation played an important role in disrupting intestinal tight junctions (Hu et al., 2013). Our results indicate that although a dietary carvacrol-thymol blend decreased the counts of Enterococcus genus and
E. coli, and thereby bacterial infection involved in inflammation, it might have limited ability to control intestinal inflammation caused by several other potential mechanisms, including intolerance to particular allergens in feeds and corticotropin releasing factor-induced mast cell activation (Smith et al., 2010).

The effect of supplementation of plant extracts and essential oil blends on performance of weaning pigs has been evaluated in several studies. Most of the studies suggested that supplementation of plant extracts and essential oil blends does not improve performance of weaning pigs, regardless of whether carvacrol or thymol is contained or not (Namkung et al., 2004; Cho et al., 2006; Kommera et al., 2006; Manzanilla et al., 2006; Nofrarias et al., 2006). For example, the dietary supplementation with the plant extract containing 5\% (w/w) carvacrol, 3\% cinnamaldehyde and 2\% capsicum oleoresin at dose of $300 \mathrm{mg} / \mathrm{kg}$ does not affect the performance of weaning pigs (Manzanilla et al., 2006; Nofrarias et al., 2006). In addition, feeding a diet supplemented with $1000 \mathrm{mg} / \mathrm{kg}$ essential oil blend containing $60 \%$ active substance (cymene, terpinene, carvacrol) also failed to improve the performance of weaning pigs (Huang et al., 2010).

In contrast, feeding the diet supplemented with $100 \mathrm{mg} / \mathrm{kg}$ essential oil blend containing 18\% thymol and cinnamaldehyde increases daily weight gain of weaning pigs compared with those fed with unsupplemented basal diet (Li et al., 2012a and 2012b; Zeng et al., 2015). Those increased performance may be caused by increased nutrient digestibility (Li et al., 2012a; Zeng et al., 2015). Moreover, all the animals in those studies are fed with experimental diet for 4 or 5 weeks, whereas only a 2-week feeding trial was performed in the current study. In fact, feeding the $100 \mathrm{mg} / \mathrm{kg}$ essential oil blend containing $18 \%$ thymol and cinnamaldehyde for 1 week does not affect the performance of weaning pigs (Li et al., 2012b). In consistence with the effect of performance, the influence of nutrient digestibility is observed after 4 or 5 weeks of feeding the $100 \mathrm{mg} / \mathrm{kg}$ essential oil blend supplemented diet (Li et al., 2012a; Zeng et al., 2015).

A previous study showed that feeding a diet supplemented with 2000 or $3000 \mathrm{mg} / \mathrm{kg}$ essential oil blend, which contains 
$60 \mathrm{mg} / \mathrm{kg}$ carvacrol and $55 \mathrm{mg} / \mathrm{kg}$ thymol, for 3 weeks, increases feed intake and average daily gain of weaning pigs compared with those fed with unsupplemented basal diet (Molnar and Bilkei, 2005). However, supplementation of the essential oil blend at dose of $1000 \mathrm{mg} / \mathrm{kg}$ to provide $60 \mathrm{mg}$ carvacrol and $55 \mathrm{mg}$ thymol/ $\mathrm{kg}$ feed, which is close to the concentration of carvacrol and thymol provided by diet in the current study, does not affect the performance of weaning pigs (Molnar and Bilkei, 2005). These results indicated that the concentration of carvacrol and thymol $>100 \mathrm{mg} / \mathrm{kg}$ and the feeding period longer than 3 weeks might improve performance of weaning pigs effectively.

It has been demonstrated that feeding a diet supplemented with an antioxidant blend (200 mg vitamin C, $100 \mathrm{mg}$ vitamin $\mathrm{E}, 450 \mathrm{mg}$ tea polyphenols, $1 \mathrm{~g}$ lipoic acid and $5 \mathrm{~g}$ microbial antioxidants fermented by Bacillus, Lactobacillus, photosynthetic bacteria and beer yeast) reduces intestinal oxidative stress and prevents intestinal villus height and villus:crypt ratio in weaning piglets (Xu et al., 2014b). Among the ingredients in the antioxidant blend used in that study, both tea polyphenols and lipoic acid exert antiinflammatory effects via inhibition of the nuclear factor- $\kappa \mathrm{B}(\mathrm{NF}-\kappa \mathrm{B})$ signaling pathway (Goraca et al., 2015; Li et al., 2015; Marinovic et al., 2015). Therefore, the antioxidant blend used in that study might also have reduced weaning-induced intestinal inflammation effectively. Considering the complex mechanism of intestinal barrier dysfunction in weaning piglets, a more complex blended dietary supplement containing antiinflammatory, antioxidative and antibacterial ingredients might enhance the intestinal health of weaning piglets more effectively.

In conclusion, the results of this study showed that weaning stress induced both intestinal oxidative stress and dysfunction of the intestinal barrier, indicated by influenced mRNA levels of tight junction proteins and activity of plasma D-lactate in piglets. Dietary supplementation with a carvacrol-thymol blend reduced weaning-induced intestinal oxidative stress, decreased the populations of Enterococcus genus and E. coli, and increased the population of Lactobacillus genus in the jejunum. However, a carvacrol-thymol blend had limited effects on weaning-induced intestinal inflammation and dysfunction of the intestinal barrier.

\section{Acknowledgments}

This study was supported by the International Scientific and Technological Cooperation Program (No. 2013DFG32510), the Special Fund for Agro-Scientific Research in the Public Interest (No. 201003060), the Hubei Provincial Creative Team Project of Agricultural Science and Technology (No. 2007-620), the Hubei Provincial Natural Science Foundation (No. 2013CFA010) and the Fundamental Research Funds for the Central Universities (2013PY047 and 2013QC004). Hong-Kui Wei, Hong-Xing Xue and Jian Peng have no conflicts of interest. The authors thank Novus International Inc. for providing NE150 ${ }^{\circledR}$. Jian Peng and Hong-Kui Wei designed the research methodology, Hong-Kui Wei and Hong-Xing Xue conducted the research, Hong-Xing
Xue analyzed the data, and Hong-Kui Wei, Hong-Xing Xue and Jian Peng wrote the paper. Jian Peng had primary responsibility for the final content. All authors read and approved the final manuscript.

\section{References}

Al-Sadi R, Boivin M and Ma T 2009. Mechanism of cytokine modulation of epithelial tight junction barrier. Frontiers in Bioscience 14, 2765-2778.

Archana PR, Nageshwar Rao B, Ballal M and Satish Rao BS 2009. Thymol, a naturally occurring monocyclic dietary phenolic compound protects Chinese hamster lung fibroblasts from radiation-induced cytotoxicity. Mutation Research/Genetic Toxicology and Environmental Mutagenesis 680, 70-77.

Campbell JM, Crenshaw JD and Polo J 2013. The biological stress of early weaned piglets. Journal of Animal Science and Biotechnology 4, 1-4.

Castillo M, Martin-Orue SM, Nofrarias M, Manzanilla EG and Gasa J 2007. Changes in caecal microbiota and mucosal morphology of weaned pigs. Veterinary Microbiology 124, 239-247.

Cho JH, Chen YJ, Min BJ, Kim HJ, Kwon OS, Shon KS, Kim IH, Kim SJ and Asamer A 2006. Effects of essential oils supplementation on growth performance, IgG concentration and fecal noxious gas concentration of weaned pigs. Asian-Australasian Journal of Animal Sciences 19, 80-85.

Degroote J, Michiels J, Claeys E, Ovyn A and De Smet S 2012. Changes in the pig small intestinal mucosal glutathione kinetics after weaning. Journal of Animal Science 90 (suppl. 4), 359-361.

Du D, Shi Y-H and Le G-W 2010. The effect of diet with different glycemic index on the redox status of duodenums in mice and its underlying mechanism. European Food Research and Technology 230, 935-941.

Franklin MA, Mathew AG, Vickers JR and Clift RA 2002. Characterization of microbial populations and volatile fatty acid concentrations in the jejunum, ileum, and cecum of pigs weaned at 17 vs 24 days of age. Journal of Animal Science 80, 2904-2910.

Goraca A, Huk-Kolega H, Kowalczyk A and Skibska B 2015. Anti-oxidative and anti-inflammatory effects of lipoic acid in rat liver. Postepy Higieny I Medycyny Doswiadczalnej 69, 270-276.

Guarda A, Rubilar JF, Miltz J and Galotto MJ 2011. The antimicrobial activity of microencapsulated thymol and carvacrol. International Journal of Food Microbiology 146, 144-150.

Gutiérrez-Fernández J, García-Armesto MR, Álvarez-Alonso R, del Valle P, de Arriaga D and Rúa J 2013. Antimicrobial activity of binary combinations of natural and synthetic phenolic antioxidants against Enterococcus faecalis. Journal of Dairy Science 96, 4912-4920.

Hou YQ, Wang L, Zhang W, Yang ZG, Ding BY, Zhu HL, Liu YL, Qiu YS, Yin $Y L$ and Wu GY 2012. Protective effects of $\mathrm{N}$-acetylcysteine on intestinal functions of piglets challenged with lipopolysaccharide. Amino Acids 43, 1233-1242.

Hu CH, Xiao K, Luan ZS and Song J 2013. Early weaning increases intestinal permeability, alters expression of cytokine and tight junction proteins, and activates mitogen-activated protein kinases in pigs. Journal of Animal Science 91, 1094-1101.

Huang Y, Yoo JS, Kim HJ, Wang Y, Chen YJ, Cho JH and Kim IH 2010. Effects of dietary supplementation with blended essential oils on growth performance, nutrient digestibility, blood profiles and fecal characteristics in weanling pigs. Asian-Australasian Journal of Animal Sciences 23, 607-613.

Huycke MM and Moore DR 2002. In vivo production of hydroxyl radical by Enterococcus faecalis colonizing the intestinal tract using aromatic hydroxylation. Free Radical Biology and Medicine 33, 818-826.

Kommera SK, Mateo RD, Neher FJ and Kim SW 2006. Phytobiotics and organic acids as potential alternatives to the use of antibiotics in nursery pig diets. Asian-Australasian Journal of Animal Sciences 19, 1784-1789.

Li GF, Fu JH, Zhao Y, Ji KQ, Luan T and Zang B 2015. Alpha-lipoic acid exerts anti-inflammatory effects on lipopolysaccharide-stimulated rat mesangial cells via inhibition of nuclear factor kB (NF-kB) signaling pathway. Inflammation 38, 510-519.

Li PF, Piao XS, Ru YJ, Han X, Xue LF and Zhang HY 2012. Effects of adding essential oil to the diet of weaned pigs on performance, nutrient utilization, immune response and intestinal health. Asian-Australasian Journal of Animal Sciences 25, 1617-1626. 
Li SY, Ru YJ, Liu M, Xu B, Péron A and Shi XG 2012. The effect of essential oils on performance, immunity and gut microbial population in weaner pigs. Livestock Science 145, 119-123.

Manzanilla EG, Nofrarias M, Anguita M, Castillo M, Perez JF, Martin-Orue SM, Kamel C and Gasa J 2006. Effects of butyrate, avilamycin, and a plant extract combination on the intestinal equilibrium of early-weaned pigs. Journal of Animal Science 84, 2743-2751.

Marinovic MP, Morandi AC and Otton R 2015. Green tea catechins alone or in combination alter functional parameters of human neutrophils via suppressing the activation of TLR-4/NF kB p65 signal pathway. Toxicology In Vitro 29, 1766-1778.

Marquardt RR, Jin L, Kim J-W, Fang L, Frohlich AA and Baidoo SK 1999. Passive protective effect of egg-yolk antibodies against enterotoxigenic Escherichia coli K88+ infection in neonatal and early-weaned piglets. FEMS Immunology \& Medical Microbiology 23, 283-288.

Mastelic J, Jerkovic I, Blazevic I, Poljak-Blazi M, Borovic S, Ivancic-Bace I, Smrecki V, Zarkovic N, Brcic-Kostic K, Vikic-Topic D and Mueller N 2008. Comparative study on the antioxidant and biological activities of carvacrol, thymol, and eugenol derivatives. Journal of Agricultural and Food Chemistry 56, 3989-3996.

Michiels J, Missotten JAM, Fremaut D, De Smet S and Dierick NA 2009. In vitro characterisation of the antimicrobial activity of selected essential oil components and binary combinations against the pig gut flora. Animal Feed Science and Technology 151, 111-127.

Molnar C and Bilkei G 2005. The influence of an oregano feed additive on production parameters and mortality of weaned piglets. Tierärztliche Praxis. Ausgabe G, Grosstiere/Nutztiere 33, 42-47.

Namkung H, Li M, Gong J, Yu H, Cottrill M and de Lange CFM 2004. Impact of feeding blends of organic acids and herbal extracts on growth performance, gut microbiota and digestive function in newly weaned pigs. Canadian Journal of Animal Science 84, 697-704.

Nofrarias M, Manzanilla EG, Pujols J, Gibert X, Majo N, Segales J and Gasa J 2006. Effects of spray-dried porcine plasma and plant extracts on intestinal morphology and on leukocyte cell subsets of weaned pigs. Journal of Animal Science 84, 2735-2742.

Padgett LE, Broniowska KA, Hansen PA, Corbett JA and Tse HM 2013. The role of reactive oxygen species and proinflammatory cytokines in type 1 diabetes pathogenesis. Year in Diabetes and Obesity 1281, 16-35.

Peace RM, Campbell J, Polo J, Crenshaw J, Russell L and Moeser A 2011. Spraydried porcine plasma influences intestinal barrier function, inflammation, and diarrhea in weaned pigs. The Journal of Nutrition 141, 1312-1317.

Rada B, Gardina P, Myers TG and Leto TL 2011. Reactive oxygen species mediate inflammatory cytokine release and EGFR-dependent mucin secretion in airway epithelial cells exposed to Pseudomonas pyocyanin. Mucosal Immunology 4, 158-171.

Safaei-Ghomi J, Ebrahimabadi AH, Djafari-Bidgoli Z and Batooli H 2009. GC/MS analysis and in vitro antioxidant activity of essential oil and methanol extracts of Thymus caramanicus Jalas and its main constituent carvacrol. Food Chemistry $115,1524-1528$

Smith F, Clark JE, Overman BL, Tozel CC, Huang JH, Rivier JEF, Blisklager AT and Moeser AJ 2010. Early weaning stress impairs development of mucosal barrier function in the porcine intestine. American Journal of PhysiologyGastrointestinal and Liver Physiology 298, G352-G363.

Sun J, Hu XL, Le GW and Shi YH 2010. Lactobacilli prevent hydroxy radical production and inhibit Escherichia coli and Enterococcus growth in system mimicking colon fermentation. Letters in Applied Microbiology 50, 264-269.

Suzuki T, Yoshinaga N and Tanabe S 2011. Interleukin-6 (IL-6) regulates claudin-2 expression and tight junction permeability in intestinal epithelium. Journal of Biological Chemistry 286, 31263-31271.

Wang JJ, Chen LX, Li P, Li XL, Zhou HJ, Wang FL, Li DF, Yin YL and Wu GY 2008. Gene expression is altered in piglet small intestine by weaning and dietary glutamine supplementation. The Journal of Nutrition 138, 1025-1032.

Wei H-K, Chen G, Wang R-J and Peng J 2015. Oregano essential oil decreased susceptibility to oxidative stress-induced dysfunction of intestinal epithelial barrier in rats. Journal of Functional Foods 18, 1191-1199.

Wijtten PJA, van der Meulen J and Verstegen MWA 2011. Intestinal barrier function and absorption in pigs after weaning: a review. British Journal of Nutrition 105, 967-981.

Wolvekamp MCJ and de Bruin RWF 1994. Diamine oxidase: an overview of historical, biochemical and functional aspects. Digestive Diseases 12, 2-14.

Xu CC, Yang SF, Zhu LH, Cai X, Sheng YS, Zhu SW and Xu JX 2014a. Regulation of $\mathrm{N}$-acetyl cysteine on gut redox status and major microbiota in weaned piglets. Journal of Animal Science 92, 1504-1511.

Xu JX, Xu CC, Chen XL, Cai X, Yang SF, Sheng YS and Wang T 2014b. Regulation of an antioxidant blend on intestinal redox status and major microbiota in early weaned piglets. Nutrition 30, 584-589.

Zeng ZK, Xu X, Zhang Q, Li P, Zhao PF, Li QY, Liu JD and Piao XS 2015. Effects of essential oil supplementation of a low-energy diet on performance, intestinal morphology and microflora, immune properties and antioxidant activities in weaned pigs. Animal Science Journal 86, 279-285.

Zhu LH, Zhao KL, Chen XL and Xu JX 2012. Impact of weaning and an antioxidant blend on intestinal barrier function and antioxidant status in pigs. Journal of Animal Science 90, 2581-2589. 